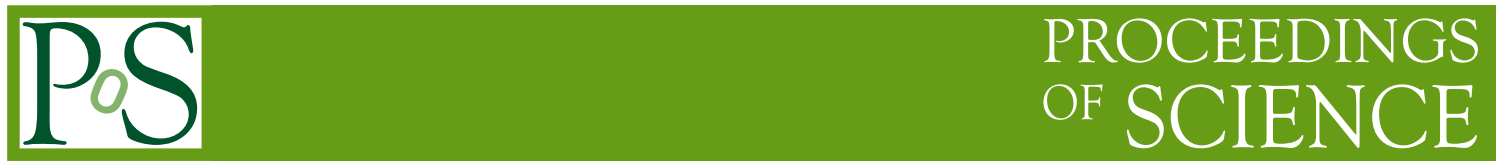

\title{
RFI Mitigation in AIPS. The New Task UVRFI
}

\section{Leonid Kogan*}

National Radio Astronomy Observatory, Socorro, New Mexico, USA

E-mail: lkogan@nrao.edu

\section{Frazer Owen}

National Radio Astronomy Observatory, Socorro, New Mexico, USA

E-mail: fowen@nrao.edu

Recently Ramana Athrea published a new algorithm ([1]) based on the difference at fringe rates of a source in the sky and ground-based RFI. His algorithm works only for ground-based and constant-amplitude RFI during a solution interval. We modified his algorithm to include a possible change of the RFI's amplitude during the solution interval and developed another algorithm based on Högbom CLEANing of the Fourier transform of the time series of the SOURCE+RFI visibilities. These algorithms allow us to mitigate RFI originating from more than one source moving with different nonzero speeds relative to the array (e.g. ground-based and satellite-based RFI). The new algorithms are implemented in AIPS ([2]) in the task UVRFI. The result of testing this task is demonstrated using the EVLA data at L band. It is also shown that self-averaging of RFI can reduce its impact on imaging even if the solution interval in the correlator is too small to allow self-averaging before imaging.

RFI mitigation workshop - RFI2010,

March 29-31, 2010

Groningen, the Netherlands

\footnotetext{
* Speaker.
} 


\section{Introduction}

Sources of RFI generally have different fringe rates than astronomical sources of interest to astronomers. This difference has been exploited by many researches to separate and excise RFI. (see for example ([3], [4]). The visibility for the given interferometer baseline, frequency channel, polarization, time is determined by the following expression:

$$
V i s_{\text {obs }}=V i s_{\text {source }} \cdot \exp j \omega_{\text {frso }} t+V i s_{r f i g r}+V i s_{r f i s a t 1} \cdot \exp j \omega_{\text {frsat } 1} t+\ldots . .
$$

where $V i s_{\text {source }}$ is the visibility of an astronomical source;

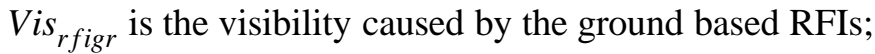

Vis $_{\text {rfisat } 1}$ is the visibility caused by RFI from satellite 1;

$\omega_{\text {frso }}$ is the fringe rate of the source, caused by earth rotation;

$\omega_{\text {frsat } 1}$ is the fringe rate of the RFIs, caused by the motion of satellite 1;

Note that the fringe rate caused by ground-based RFI is equal to zero, because the ground-based RFIs do not move relatively the ground-based array.

Practically any array correlator multiplies the observed visibility by the fringe stopping complex exponent $\exp -j \omega_{f r s o} t$. As a result the source fringes are stopped but the ground-based RFI is rotated at that rate and the correlator output visibility can be described by the following expression:

$$
V i s_{\text {cor }}=V i s_{\text {source }}+V i s_{\text {rfigr }} \cdot \exp -j \omega_{f r s o} t+V i s_{r f i s a t ~} \cdot \exp j\left(\omega_{\text {frsat } 1}-\omega_{f r s o}\right) t+\ldots
$$

The problem we need to solve is formulated as:

Given a correlator output time series for a given baseline resulting from astronomical sources and RFI during some solution interval, our goal is to find the astronomical source visibility during that solution interval!

\section{Athrea's approach to the problem}

Athrea ([1]) considered RFI which is caused only by ground-based RFI. Therefore, the trajectory (in time) of the correlator output in the complex plane will be a circle with radius equal to the

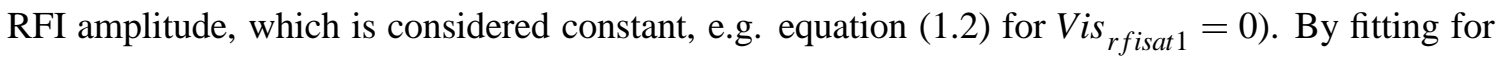
these three parameters, the resulting coordinates of the circle center are the solution for the source complex visibility without RFI.

\section{How good are the circles in practice?}

In this section we look at the quality of the "circles" using the EVLA data at L band, kindly provided by Michael Rupen. The data are the result of several minutes observation of 3C 345 by the EVLA in the D configuration. The new EVLA WIDAR correlator was used to obtain the data with sampling at time of 0.1 second and 256 frequency channels. In figure (1) we show a plot of the frequency spectrum for one baseline and polarization during one time interval of 10 seconds. The 


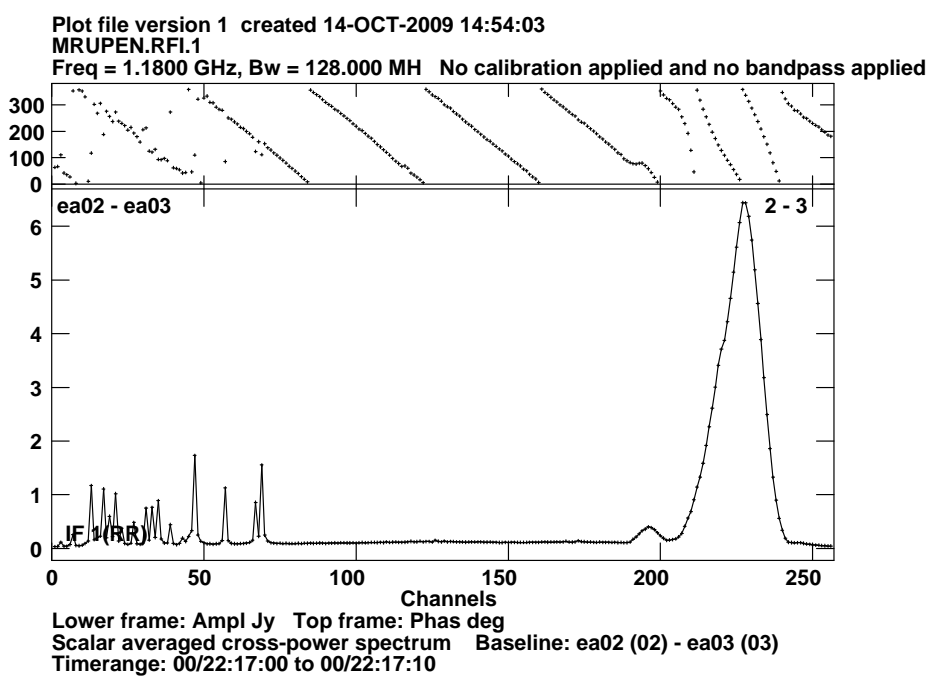

Figure 1: The visibility spectrum for one baseline of the EVLA L-band data

central part of the spectrum (free of RFI) shows good behavior of both amplitude and phase. The left part of the spectrum is full of spikes caused by a radar. The right part of the spectrum has very strong RFI caused by a group of satellites. The plot in the left top corner of the figure 2 shows the trajectory of the complex visibility at channel 224 during 10 seconds (100 points), which would be expected to be a circle in the ideal case. This 'circle' looks rather like a spiral. We call this a "circle" since it is the best example of a quasi-circle and the other "circles" appear to be much worse. The first five plots correspond to the satellite RFI (the same channel 224, different 10s time intervals). The sixth plot does not show a circle. So, looking at this set of plots we can conclude that the concept of circles may be used to mitigate RFI only in the special case of ground-based RFI, when the RFI amplitude is really constant during the solution interval.

Two reasons of the RFI amplitude variability can be offered:

1. The variable signal levels broadcast by the satellites and

2. Even if the satellites were stationary in the sky, the array antennas track the astronomical source and thus the antenna sidelobes sweep across the satellite position, modulating the strength of the RFI.

\section{The new AIPS task UVRFI}

The new AIPS task UVRFI offers the following two algorithms to mitigate RFI:

1. 'CIRC' a l'a Athrea

A spiral with four unknown parameters (initial radius, linear increment of the radius, and two coordinates of the center) is fitted to the data using the non-linear-least-square-method. The two coordinates of the center are used as a solution for the astronomical source visibility, free of RFI

2. 'CEXP' 

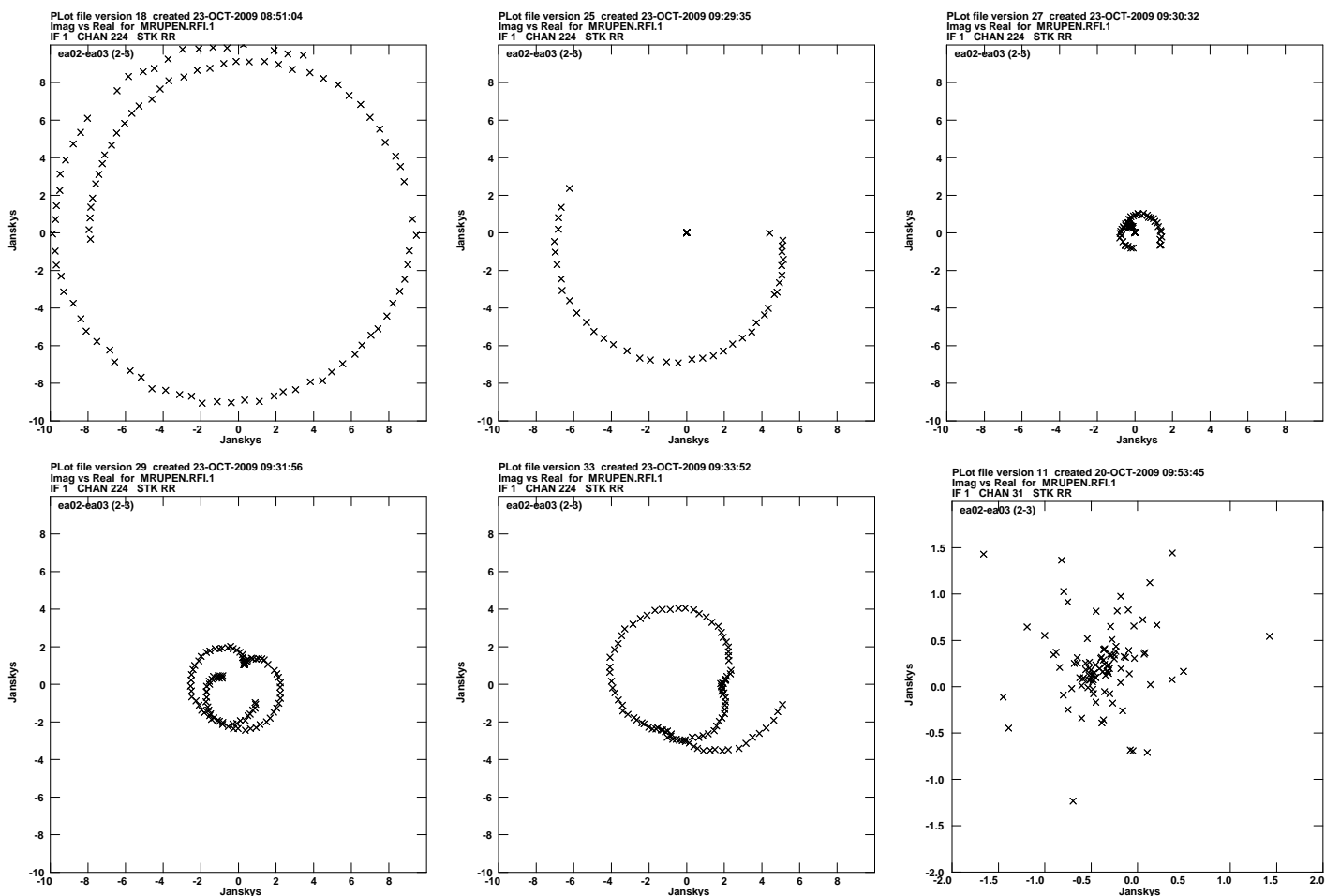

Figure 2: Shapes of the "circles" for the L-band EVLA data

This model is represented by the sum of several spectral components with complex amplitudes:

$$
V i s_{\text {cor }}=V i s_{\text {source }}+R F I_{1} \cdot \exp j \omega_{1} t+R F I_{2} \cdot \exp j \omega_{2} t+\ldots
$$

A simple, one dimensional, version of Högbom CLEAN algorithm is used to fit complex delta functions to the Fourier transform of the observed visibility time-series during each solution interval. The final solution is the value of the cleaned Fourier transform at zero frequency. No CLEANing is allowed at zero frequency to prevent the subtraction of the signal itself. Additionally, UVRFI flags the RFI caused by a radar using the fact that radar RFI on the frequency axis looks like a set of delta functions. See the left part of the figure 1 for example.

\section{Test of the task UVRFI at L band using the EVLA data}

In figure 3 we compare the UVRFI ('CEXP' option) and the AIPS task UVAVG results. The two plots show advantage of the UVRFI output (right plot): the radar RFIs are flagged completely; the satellite RFI is lower by factor 3-4. We should note that the vector averaging itself suppresses the RFI by self-averaging but not as much as with UVRFI. So the comparison could be even more in favor of UVRFI if the less averaging was done in UVAVG. In Figure 4 we compare the images using the vector averaging (UVAVG) and task UVRFI ('CEXP'). The image at the right plot (UVRFI) is obviously better! 

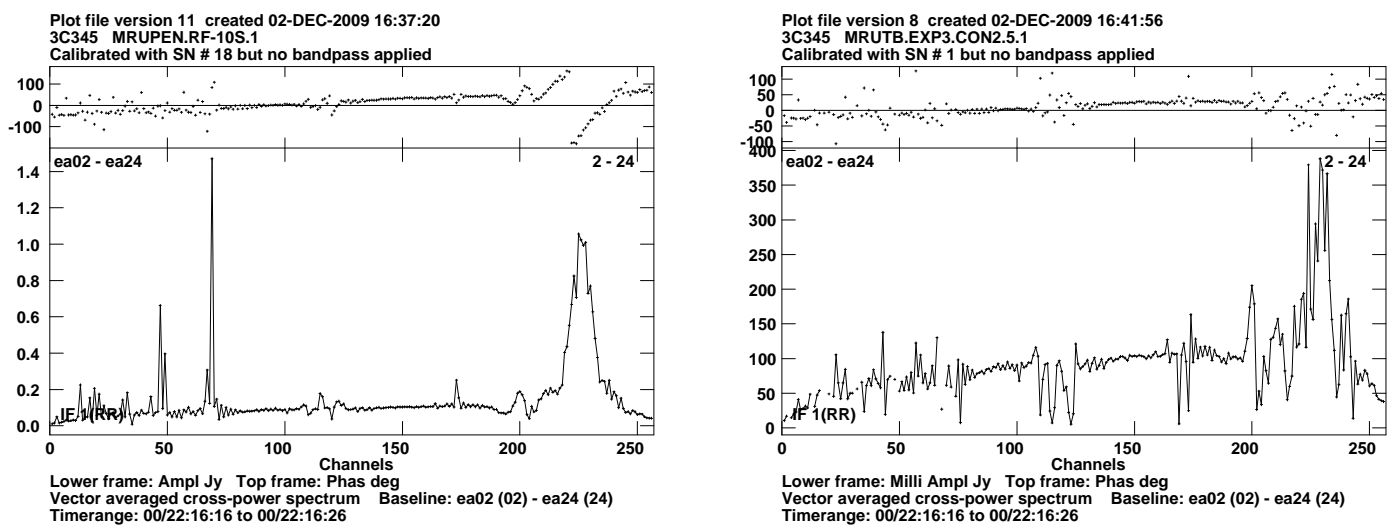

Figure 3: The visibility spectrum for one baseline of the EVLA L-band data. The left plot is the output of the AIPS task UVAVG (vector averaging in 10s). The right plot is the output of UVRFI task. OPTYPE = 'CEXP'. Solution interval $=10 \mathrm{~s}$
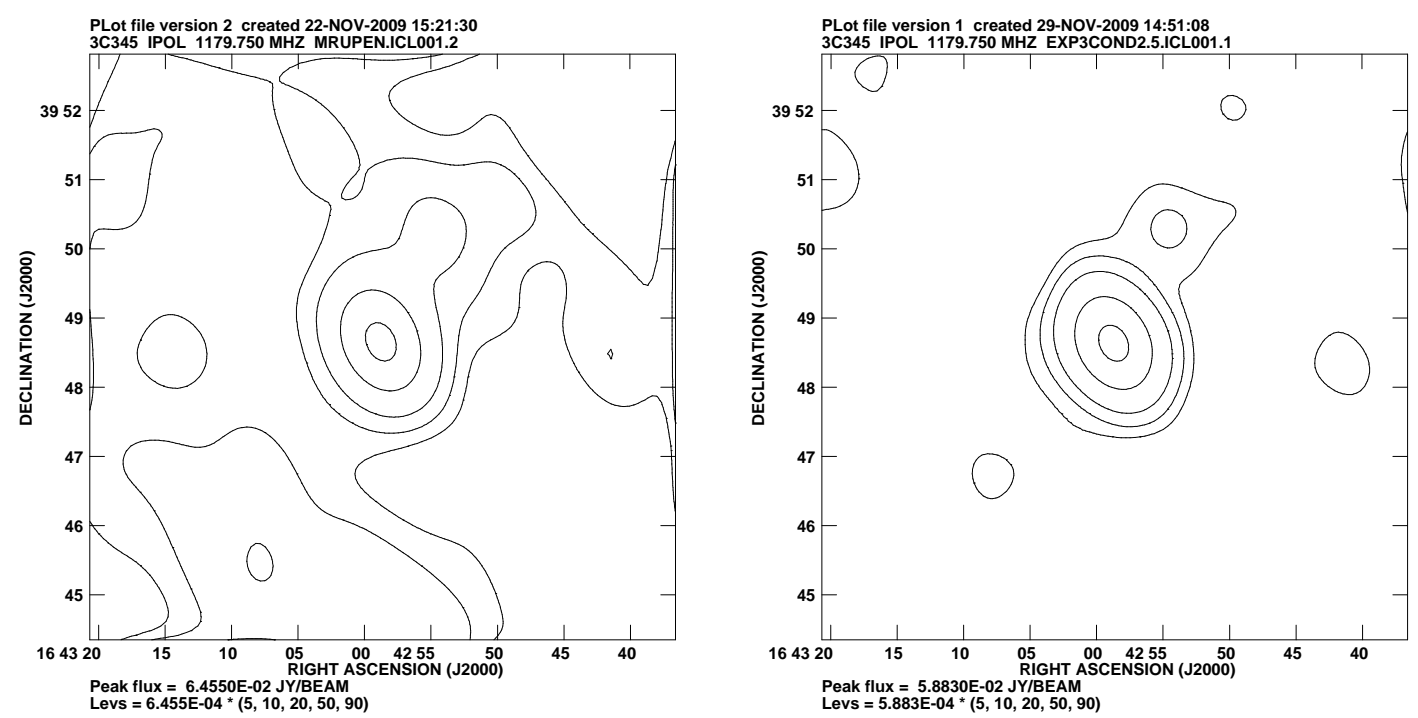

Figure 4: Comparison of the images for EVLA L-band data. The left plot is the image after UVAVG (vector averaging in 10s). The right plot is the image after UVRFI task. OPTYPE $=$ 'CEXP'. Solution interval $=$ $10 \mathrm{~s}$

\section{Self-averaging of the RFI in process of imaging.}

As we discussed previously, the visibility caused by the ground-based RFI produces a 'circle' in the complex plane. Therefore RFI can be self-averaged during vector averaging in the correlator. The effect of this self-averaging can be estimated by number of periods of the fringe rate in the correlator solution interval. At low frequency, the fringe period can be large in comparison with the correlator solution interval, and therefore the self-averaging of RFI in the correlator will not be so effective. The same argument was used by R. Athrea ([1]). He wrote: "It is often claimed that interferometric fringe stopping itself washes out RFI, but this is not entirely appropriate for low frequency array." We saw confirmation of this statement by comparing the vector averaging with 
different averaging times (AIPS task UVAVG). It might be expected that the impact of RFI would be much higher for smaller averaging times. However, the quality of the images using different averaging times is similar! The explanation of this effect is that the griding step in the imaging carries out the averaging in some cases. The following equation elucidates this. If the visibility caused by RFI is described by the following equation:

$$
R F I=A \cdot \exp j \omega_{f r} t_{i}
$$

then the relevant dirty map $D M_{r f i}$ is equal:

$$
D M_{r f i}=A \cdot \sum_{i} \exp j \omega_{f r} t_{i} \cdot \exp j 2 \pi\left(U_{i} l+V_{i} m\right)
$$

If $U_{i}, V_{i}$ are 'constant' inside of the time interval $T_{i m a g}$, then

$$
D M_{r f i}=A \cdot \sum_{k} \exp j 2 \pi\left(U_{k} l+V_{k} m\right) \cdot \sum_{i} \exp j \omega_{f r} t_{i, k}
$$

where $\mathrm{i}$ is the preaverage time interval number;

$\mathrm{k}$ is the time interval of $T_{\text {imag }}$ number

Therefore the effect of self-averaging of RFI may not be limited by the correlator averaging interval but rather by the grid cell size for the FFT used for imaging, where both $U$ and $V$ are effectively constant.

\section{Conclusions}

The new AIPS task UVRFI uses the two algorithms to mitigate RFI:

CIRC function, based on modification of Ramana Athrea algorithm, fits a spiral to the observed visibility curve in the complex plane.

CEXP function subtracts a set of the complex exponential delta functions representing RFIs, using a simple CLEAN algorithm applied to the Fourier transform of the complex visibility time-series. The second algorithm demonstrates the better result for the two datasets we studied and allows us to mitigate more than one source of RFI (e.g. ground-based, satellite-based).

The utility of RFI mitigation algorithm is complicated by the non-circular nature of the RFI in the complex plane.

In some cases, the effect of RFI may be reduced by self-averaged during imaging.

\section{References}

[1] Ramana Athrea, A New Approach to Mitigation of Radio Frequency Interference in Interferometric Data, Astrophysical Journal, vol 696, May 2009, p 885

[2] NRAO Astronomical Image Processing System

[3] T.J. Cornwell, R.A. Perley, K. Golap, S. Bhatnagar, RFI excision in synthesis imaging without a reference signal, EVLA memo 86, NRAO, December 2004.

[4] R.A. Perley, T.J. Cornwell, Removing RFI through Astronomical Image Processing, EVLA memo 61, NRAO, July 2003. 